\title{
A positive fixed point theorem with applications to systems of Hammerstein integral equations
}

\author{
Alberto Cabada', José Ángel Cid² and Gennaro Infante ${ }^{3 *}$
}

"Correspondence:
gennaro.infante@unical.it
3Dipartimento di Matematica e
Informatica, Università della
Calabria, Arcavacata di Rende,
Cosenza, 87036, Italy
Full list of author information is
available at the end of the article

${ }^{\text {*Correspondence: }}$

gennaro.infante@unical.it

Dipartimento di Matematica

Calabria, Arcavacata di Rende,

Full list of author information is

available at the end of the article

\begin{abstract}
We present new criteria on the existence of fixed points that combine some monotonicity assumptions with the classical fixed point index theory. As an illustrative application, we use our theoretical results to prove the existence of positive solutions for systems of nonlinear Hammerstein integral equations. An example is also presented to show the applicability of our results.

MSC: Primary 47H10; secondary 34B10; 34B18; 45G15; 47H30
\end{abstract}

Keywords: cone; boundary value problem; fixed point index; positive solution; nonlocal boundary condition; system

\section{Introduction}

In this manuscript we pursue the line of research developed in the recent papers [1-5] in order to deal with fixed point theorems on cones that mix monotonicity assumptions and conditions in one boundary, instead of imposing conditions on two boundaries as in the celebrated cone compression/expansion fixed point theorem of Krasnosel'skii. In order to do this we employ the well-known monotone iterative method, combined with the classical fixed point index. In Section 2 we prove two results concerning non-decreasing and non-increasing operators in a shell, in presence of an upper or of a lower solution; in Remark 2.4 we present a comparison with previous results in this direction.

In [3] Cid et al., in order to show the existence of positive solutions of the fourth-order boundary value problem (BVP)

$$
\begin{aligned}
& u^{(4)}=\lambda g(t) f(u), \quad t \in(0,1), \\
& u(0)=u(1)=0=u^{\prime \prime}(0)=u^{\prime \prime}(1),
\end{aligned}
$$

where $\lambda>0$, studied the associated Hammerstein integral equation

$$
u(t)=\lambda \int_{0}^{1} k(t, s) g(s) f(u(s)) d s,
$$

(c) 2014 Cabada et al.; licensee Springer. This is an Open Access article distributed under the terms of the Creative Commons Attribution License (http://creativecommons.org/licenses/by/4.0), which permits unrestricted use, distribution, and reproduction in any medium, provided the original work is properly credited. 
where $k$ is precisely the Green's function associated to the BVP (1.1). Having defined the constant

$$
\gamma^{*}=\max _{t \in[0,1]} \int_{0}^{1} k(t, s) g(s) d s
$$

the main result in [3], regarding the BVP (1.1), is the following.

Theorem 1.1 Assume that $\lim _{s \rightarrow \infty} \frac{f(s)}{s}=+\infty$ and there exists $B \in[0,+\infty]$ such that $f$ is non-decreasing on $[0, B)$. If

$$
0<\lambda<\sup _{s \in(0, B)} \frac{s}{\gamma^{*} f(s)}
$$

(with the obvious meaning when $f(s)=0$ ), then the BVP (1.1) has at least a positive solution.

Note that the above theorem is valid for a specific Green's function. On the other hand the existence of nonnegative solutions for systems of Hammerstein integral equations has been widely studied; see for example [6-22] and references therein. In Section 3 we give an extension of Theorem 1.1 to the context of systems of Hammerstein integral equations of the type

$$
\begin{aligned}
& u_{1}(t)=\lambda_{1} \int_{a}^{b} k_{1}(t, s) g_{1}(s) f_{1}\left(u_{1}(s), u_{2}(s)\right) d s, \\
& u_{2}(t)=\lambda_{2} \int_{a}^{b} k_{2}(t, s) g_{2}(s) f_{2}\left(u_{1}(s), u_{2}(s)\right) d s,
\end{aligned}
$$

providing, under suitable assumptions on the kernels and the nonlinearities, the existence of a positive solution.

In order to show the applicability of our results, we discuss the following system of second-order ODEs, subject to local and nonlocal boundary conditions, which generates two different kernels:

$$
\begin{aligned}
& u_{1}^{\prime \prime}(t)+\lambda_{1} f_{1}\left(u_{1}(t), u_{2}(t)\right)=0, \quad t \in(0,1), \\
& u_{2}^{\prime \prime}(t)+\lambda_{2} f_{2}\left(u_{1}(t), u_{2}(t)\right)=0, \quad t \in(0,1), \\
& u_{1}^{\prime}(0)=0, \quad u_{1}(1)+u_{1}^{\prime}(1)=0, \\
& u_{2}^{\prime}(0)=0, \quad u_{2}(1)-\xi u_{2}(\eta)=0, \quad \eta \in(0,1), 0<\xi<1,
\end{aligned}
$$

computing all the constants that occur in our theory. We also prove that the system (1.4) has a solution for every $\lambda_{1}, \lambda_{2}>0$. A similar result has been proven recently, in the context of one equation subject to nonlinear boundary conditions, by Goodrich [23].

\section{Two fixed point theorems in cones}

A subset $K$ of a real Banach space $X$ is a cone if it is closed, $K+K \subset K, \lambda K \subset K$ for all $\lambda \geq 0$, and $K \cap(-K)=\{\theta\}$. A cone $K$ defines the partial ordering in $X$ given by

$x \preceq y$ if and only if $y-x \in K$. 
We reserve the symbol ' $\leq$ ' for the usual order on the real line. For $x, y \in X$, with $x \preceq y$, we define the ordered interval

$$
[x, y]=\{z \in X: x \preceq z \preceq y\} .
$$

The cone $K$ is normal if there exists $d>0$ such that for all $x, y \in X$ with $0 \preceq x \preceq y$ then $\|x\| \leq d\|y\|$.

We denote the closed ball of center $x_{0} \in X$ and radius $r>0$ as

$$
B\left[x_{0}, r\right]=\left\{x \in X:\left\|x-x_{0}\right\| \leq r\right\},
$$

and the intersection of the cone with the open ball centered at the origin and radius $r>0$ as

$$
K_{r}=K \cap\{x \in X:\|x\|<r\} .
$$

We recall a well-known result of fixed point theory, known as the monotone iterative method (see, for example, [24, Theorem 7.A] or [25]).

Theorem 2.1 Let $N$ be a real Banach space with normal order cone K. Suppose that there exist $\alpha \leq \beta$ such that $T:[\alpha, \beta] \subset N \rightarrow N$ is a completely continuous monotone nondecreasing operator with $\alpha \leq T \alpha$ and $T \beta \leq \beta$. Then $T$ has a fixed point and the iterative sequence $\alpha_{n+1}=T \alpha_{n}$, with $\alpha_{0}=\alpha$, converges to the greatest fixed point of $T$ in $[\alpha, \beta]$, and the sequence $\beta_{n+1}=T \beta_{n}$, with $\beta_{0}=\beta$, converges to the smallest fixed point of $T$ in $[\alpha, \beta]$.

In the next proposition we recall the main properties of the fixed point index of a completely continuous operator relative to a cone, for more details see [26, 27]. In the sequel the closure and the boundary of subsets of $K$ are understood to be relative to $K$.

Proposition 2.2 Let $D$ be an open bounded set of $X$ with $0 \in D_{K}$ and $\bar{D}_{K} \neq K$, where $D_{K}=$ $D \cap K$. Assume that $T: \bar{D}_{K} \rightarrow K$ is a completely continuous operator such that $x \neq T x$ for $x \in \partial D_{K}$. Then the fixed point index $i_{K}\left(T, D_{K}\right)$ has the following properties:

(i) If there exists $e \in K \backslash\{0\}$ such that $x \neq T x+\lambda$ e for all $x \in \partial D_{K}$ and all $\lambda>0$, then $i_{K}\left(T, D_{K}\right)=0$.

For example (i) holds if $T x \npreceq x$ for $x \in \partial D_{K}$.

(ii) If $\|T x\| \geq\|x\|$ for $x \in \partial D_{K}$, then $i_{K}\left(T, D_{K}\right)=0$.

(iii) If $T x \neq \lambda x$ for all $x \in \partial D_{K}$ and all $\lambda>1$, then $i_{K}\left(T, D_{K}\right)=1$.

For example (iii) holds if either $T x \nsucceq x$ for $x \in \partial D_{K}$ or $\|T x\| \leq\|x\|$ for $x \in \partial D_{K}$.

(iv) Let $D^{1}$ be open in $X$ such that $\overline{D^{1}} \subset D_{K}$. If $i_{K}\left(T, D_{K}\right)=1$ and $i_{K}\left(T, D_{K}^{1}\right)=0$, then $T$ has a fixed point in $D_{K} \backslash \overline{D_{K}^{1}}$. The same holds if $i_{K}\left(T, D_{K}\right)=0$ and $i_{K}\left(T, D_{K}^{1}\right)=1$.

We state our first result on the existence of non-trivial fixed points.

Theorem 2.3 Let $X$ be a real Banach space, $K$ a normal cone with normal constant $d \geq 1$ and nonempty interior (i.e. solid) and $T: K \rightarrow K$ a completely continuous operator.

Assume that

(1) there exist $\beta \in K$, with $T \beta \preceq \beta$, and $R>0$ such that $B[\beta, R] \subset K$, 
(2) the map $T$ is non-decreasing in the set

$$
\mathcal{P}=\left\{x \in K: x \preceq \beta \text { and } \frac{R}{d} \leq\|x\|\right\},
$$

(3) there exists a (relatively) open bounded set $V \subset K$ such that $i_{K}(T, V)=0$ and either $\bar{K}_{R} \subset V$ or $\bar{V} \subset K_{R}$.

Then the map $T$ has at least one non-zero fixed point $x_{1}$ in $K$ such that

$$
\text { either belongs to } \mathcal{P} \text { or belongs to } \begin{cases}V \backslash \bar{K}_{R}, & \text { in case } \bar{K}_{R} \subset V, \\ K_{R} \backslash \bar{V}, & \text { in case } \bar{V} \subset K_{R} .\end{cases}
$$

Proof Since $B[\beta, R] \subset K$, if $x \in K$ with $\|x\|=R$, then $x \preceq \beta$.

Suppose first that we can choose $\alpha \in K$ with $\|\alpha\|=R$ and $T \alpha \succeq \alpha$. Since $\alpha \preceq \beta$ and due to the normality of the cone $K$ we have $[\alpha, \beta] \subset \mathcal{P}$, which implies that $T$ is non-decreasing on $[\alpha, \beta]$. Then we can apply the Theorem 2.1 to ensure the existence of a fixed point of $T$ on $[\alpha, \beta]$, which, in particular, is a non-trivial fixed point.

Now suppose that such $\alpha$ does not exist. Thus $T x \nsucceq x$ for all $x \in K$ with $\|x\|=R$, which by Proposition 2.2(iii) implies that $i_{K}\left(T, K_{R}\right)=1$. Since, by assumption, $i_{K}(T, V)=0$ we get the existence of a non-trivial fixed point $x_{1}$ belonging to the set $V \backslash \bar{K}_{R}$ (when $\bar{K}_{R} \subset V$ ) or to the $K_{R} \backslash \bar{V}$ (when $\bar{V} \subset K_{R}$ ).

Remark 2.4 We note that we can use either Proposition 2.2(i), or Proposition 2.2(ii), in order to check the assumption (3) in Theorem 2.3. We also stress that $\mathcal{P}$ is contained in the set $\left\{x \in K: \frac{R}{d} \leq\|x\| \leq d\|\beta\|\right\}$. Therefore Theorem 2.3 is a genuine generalization of the previous fixed point theorems obtained in [1-4]. Moreover, we show in the applications that in many cases it is useful to apply Theorem 2.3 with a set $V$ different from $K_{r}$.

We observe that, following some ideas introduced in [2, Theorem 2.1], it is possible to modify the assumptions of Theorem 2.3 in order to deal with non-increasing operators. The next result describes precisely this situation.

Theorem 2.5 Let $X$ be a real Banach space, $K$ a cone with nonempty interior (i.e. solid) and $T: K \rightarrow K$ a completely continuous operator.

Assume that

( $\left.1^{\prime}\right)$ there exist $\alpha \in K$, with $T \alpha \preceq \alpha$, and $0<R<\|\alpha\|$ such that $B[\alpha, R] \subset K$,

$\left(2^{\prime}\right)$ the map $T$ is non-increasing in the set

$$
\widetilde{\mathcal{P}}=\{x \in K: R \leq\|x\| \leq\|\alpha\|\},
$$

$\left(3^{\prime}\right)$ there exists a (relatively) open bounded set $V \subset K$ such that $i_{K}(T, V)=1$ and either $\bar{K}_{R} \subset V$ or $\bar{V} \subset K_{R}$.

Then the map $T$ has at least one non-zero fixed point such that

$$
\text { either belongs to } \widetilde{\mathcal{P}} \text { or belongs to } \begin{cases}V \backslash \bar{K}_{R}, & \text { in case } \bar{K}_{R} \subset V, \\ K_{R} \backslash \bar{V}, & \text { in case } \bar{V} \subset K_{R} .\end{cases}
$$


Proof Let $x \in K$ be such that $\|x\|=R$. Then by $\left(1^{\prime}\right)$ we have $x \preceq \alpha$ and since $x, \alpha \in \widetilde{\mathcal{P}}$ it follows from $\left(2^{\prime}\right)$ that

$$
T x \succeq T \alpha \succeq \alpha \succeq x .
$$

Now, if for some $x \in \partial K_{R}$ is the case that $T x \preceq x$ then we are done. If not, $T x \npreceq x$ for all $x \in \partial K_{R}$ which by Proposition 2.2 implies that $i_{K}\left(T, K_{R}\right)=0$. This result together with (3') gives the existence of a non-zero fixed point with the desired localization property.

\section{An application to a system of Hammerstein integral equations}

We now apply the results of the previous section in order to prove the existence of positive solutions of the system of integral equations

$$
\begin{aligned}
& u_{1}(t)=\lambda_{1} \int_{a}^{b} k_{1}(t, s) g_{1}(s) f_{1}\left(u_{1}(s), u_{2}(s)\right) d s:=T_{1}\left(u_{1}, u_{2}\right)(t), \\
& u_{2}(t)=\lambda_{2} \int_{a}^{b} k_{2}(t, s) g_{2}(s) f_{2}\left(u_{1}(s), u_{2}(s)\right) d s:=T_{2}\left(u_{1}, u_{2}\right)(t),
\end{aligned}
$$

where we assume the following assumptions:

$\left(\mathrm{H}_{1}\right) \lambda_{i}>0$, for $i=1,2$.

$\left(\mathrm{H}_{2}\right) k_{i}:[a, b] \times[a, b] \rightarrow[0,+\infty)$ is continuous, for $i=1,2$.

$\left(\mathrm{H}_{3}\right) g_{i}:[a, b] \rightarrow[0,+\infty)$ is continuous, $g_{i}(s)>0$ for all $s \in[a, b]$, for $i=1,2$.

$\left(\mathrm{H}_{4}\right) f_{i}:[0,+\infty) \times[0,+\infty) \rightarrow[0,+\infty)$ is continuous, for $i=1,2$.

$\left(\mathrm{H}_{5}\right)$ There exist continuous functions $\Phi_{i}:[a, b] \rightarrow[0,+\infty)$ and constants $0<c_{i}<1, a \leq$ $a_{i}<b_{i} \leq b$ such that for every $i=1,2$,

$$
k_{i}(t, s) \leq \Phi_{i}(s) \quad \text { for } t, s \in[a, b] \quad \text { and } \quad c_{i} \cdot \Phi_{i}(s) \leq k_{i}(t, s) \quad \text { for } t \in\left[a_{i}, b_{i}\right] \text { and } s \in[a, b] \text {, }
$$

and

$$
\gamma_{i, *}:=\min _{t \in\left[a_{i}, b_{i}\right]} \int_{a_{i}}^{b_{i}} g_{i}(s) k_{i}(t, s) d s>0
$$

We work in the space $C[a, b] \times C[a, b]$ endowed with the norm

$$
\left\|\left(u_{1}, u_{2}\right)\right\|:=\max \left\{\left\|u_{1}\right\|_{\infty},\left\|u_{2}\right\|_{\infty}\right\}
$$

where $\|w\|_{\infty}:=\max _{t \in[a, b]}|w(t)|$.

Set $c=\min \left\{c_{1}, c_{2}\right\}$ and let us define

$$
\tilde{K}_{i}:=\left\{w \in C[a, b]: w(t) \geq 0 \text { for all } t \in[a, b] \text { and } \min _{t \in\left[a_{i}, b_{i}\right]} w(t) \geq c\|w\|_{\infty}\right\}
$$

and consider the cone $K$ in $C[a, b] \times C[a, b]$ defined by

$$
K:=\left\{\left(u_{1}, u_{2}\right) \in \tilde{K}_{1} \times \tilde{K}_{2}\right\}
$$

which is a normal cone with $d=1$. 
Under our assumptions it is routine to check that the integral operator

$$
T\left(u_{1}, u_{2}\right)(t):=\left(T_{1}\left(u_{1}, u_{2}\right)(t), T_{2}\left(u_{1}, u_{2}\right)(t)\right)
$$

leaves $K$ invariant and is completely continuous.

Now we present our main result concerning the existence of positive solutions for the system (3.1).

Theorem 3.1 Assume that the assumptions $\left(\mathrm{H}_{1}\right)-\left(\mathrm{H}_{5}\right)$ hold and moreover:

$\left(\mathrm{H}_{6}\right)$ There exist constants $B_{1}, B_{2}>0$ such that for every $i=1,2, f_{i}(\cdot, \cdot)$ is non-decreasing on $\left[0, B_{1}\right] \times\left[0, B_{2}\right]$ (that is, if $\left(u_{1}, u_{2}\right),\left(v_{1}, v_{2}\right) \in \mathbb{R}^{2}$ with $0 \leq u_{i} \leq v_{i} \leq B_{i}$ for $i=1,2$, then $f_{i}\left(u_{1}, u_{2}\right) \leq f_{i}\left(v_{1}, v_{2}\right)$ for $\left.i=1,2\right)$.

$\left(\mathrm{H}_{7}\right)$ For every $M>0$ there exists $\rho=\rho(M)>0$ such that, for every $i=1,2$,

$$
\begin{aligned}
& \inf \left\{\frac{f_{1}(u, v)}{\rho}:(u, v) \in[\rho, \rho / c] \times[0, \rho / c]\right\}>M, \\
& \inf \left\{\frac{f_{2}(u, v)}{\rho}:(u, v) \in[0, \rho / c] \times[\rho, \rho / c]\right\}>M .
\end{aligned}
$$

Then the system (3.1) has at least one positive solution in $K$ provided that

$$
0<\lambda_{i}<\sup _{r_{1} \in\left(0, B_{1}\right), r_{2} \in\left(0, B_{2}\right)} \frac{(1-c) r_{i}}{f_{i}\left(r_{1}, r_{2}\right) \gamma_{i}^{*}}
$$

where

$$
\gamma_{i}^{*}:=\max _{t \in[a, b]} \int_{a}^{b} g_{i}(s) k_{i}(t, s) d s>0, \quad \text { for } i=1,2 .
$$

Proof Due to (3.2) we can fix $\beta_{i} \in\left(0, B_{i}\right), i=1,2$, such that

$$
\beta_{i}-\lambda_{i} \gamma_{i}^{*} f_{i}\left(\beta_{1}, \beta_{2}\right)>c \beta_{i}, \quad i=1,2
$$

On the other hand, for $M>\max \left\{\frac{1}{\lambda_{1} \gamma_{1, *}}, \frac{1}{\lambda_{2} \gamma_{2, *}}\right\}$ let $\rho=\rho(M)>0$ as in $\left(\mathrm{H}_{7}\right)$ and fix $R<$ $\min \left\{\frac{1-c}{1+c} \cdot \beta_{1}, \frac{1-c}{1+c} \cdot \beta_{2}, \rho\right\}$.

Let us check that the assumptions of Theorem 2.3 are satisfied with

$$
\beta(t)=\left(\beta_{1}, \beta_{2}\right) \quad \text { for all } t \in[a, b]
$$

and

$$
V=\left\{\left(u_{1}, u_{2}\right) \in K: \min _{t \in\left[a_{1}, b_{1}\right]} u_{1}(t)<\rho \text { and } \min _{t \in\left[a_{2}, b_{2}\right]} u_{2}(t)<\rho\right\} .
$$

Claim 1. $B[\beta, R] \subset K$ and $T \beta \preceq \beta$.

Since $\beta$ is constant and $R<\min \left\{\frac{1-c}{1+c} \cdot \beta_{1}, \frac{1-c}{1+c} \cdot \beta_{2}\right\}$ a direct computation shows that $B[\beta, R] \subset K$. Now, from (3.3) it follows for each $t \in[a, b]$ and $i=1,2$

$$
\left[T_{i} \beta\right](t)=\lambda_{i} \int_{a}^{b} k_{i}(t, s) g_{i}(s) f_{i}\left(\beta_{1}, \beta_{2}\right) d s \leq \lambda_{i} \gamma_{i}^{*} f_{i}\left(\beta_{1}, \beta_{2}\right)<\beta_{i}
$$


Moreover, since $\left\|\beta_{i}-T_{i} \beta\right\|_{\infty} \leq \beta_{i}, i=1,2$, and taking into account (3.3) we have for $t \in\left[a_{i}, b_{i}\right]$ and $i=1,2$,

$$
\begin{aligned}
\beta_{i}-\left[T_{i} \beta\right](t) & =\beta_{i}-\lambda_{i} \int_{a}^{b} k_{i}(t, s) g_{i}(s) f_{i}\left(\beta_{1}, \beta_{2}\right) d s \\
& \geq \beta_{i}-\lambda_{i} \gamma_{i}^{*} f_{i}\left(\beta_{1}, \beta_{2}\right)>c \beta_{i} \geq c\left\|\beta_{i}-T_{i} \beta\right\|_{\infty} .
\end{aligned}
$$

As a consequence, we have $T \beta \preceq \beta$, and the claim is proven.

Claim 2. $T$ is non-decreasing on the set $\{x \in K: x \preceq \beta\}$.

Let $u=\left(u_{1}, u_{2}\right), v=\left(v_{1}, v_{2}\right) \in K$ be such that $0 \leq u_{i}(t) \leq v_{i}(t) \leq \beta_{i}$ for all $t \in[a, b]$ and $i=1$, 2. Since $f$ is non-decreasing in $\left[0, \beta_{1}\right] \times\left[0, \beta_{2}\right]$ we have for all $t \in[a, b]$ and $i=1,2$,

$$
\left[T_{i} v\right](t)-\left[T_{i} u\right](t)=\lambda_{i} \int_{a}^{b} k_{i}(t, s) g_{i}(s)\left[f_{i}(v(s))-f_{i}(u(s))\right] d s \geq 0 .
$$

Moreover, for all $t \in\left[a_{i}, b_{i}\right], r \in[0,1]$ and $i=1,2$,

$$
\begin{aligned}
{\left[T_{i} v\right](t)-\left[T_{i} u\right](t) } & =\lambda_{i} \int_{a}^{b} k_{i}(t, s) g_{i}(s)\left[f_{i}(v(s))-f_{i}(u(s))\right] d s \\
& \geq \lambda_{i} \int_{a}^{b} c \Phi_{i}(s) g_{i}(s)\left[f_{i}(v(s))-f_{i}(u(s)] d s\right. \\
& \geq c \lambda_{i} \int_{a}^{b} k_{i}(r, s) g_{i}(s)\left[f_{i}(v(s))-f_{i}(u(s))\right] d s \\
& =c\left(\left[T_{i} v\right](r)-\left[T_{i} u\right](r)\right),
\end{aligned}
$$

therefore $\min _{t \in\left[a_{i}, b_{i}\right]}\left(\left[T_{i} v\right](t)-\left[T_{i} u\right](t)\right) \geq c\left\|T_{i} v-T_{i} u\right\|_{\infty}, i=1,2$, so $T u \preceq T v$, and since $\mathcal{P} \subset\{x \in K: x \preceq \beta\}, T$ is also non-decreasing on $\mathcal{P}$.

Claim 3. $\bar{K}_{R} \subset V$ and $i_{K}(T, V)=0$.

Firstly, note that since $R<\rho$ then we have $\bar{K}_{R} \subset K_{\rho} \subset V$.

Now let $e(t) \equiv 1$ for $t \in[a, b]$. Then $(e, e) \in K$ and we are going to prove that

$$
\left(u_{1}, u_{2}\right) \neq T\left(u_{1}, u_{2}\right)+\mu(e, e) \quad \text { for }\left(u_{1}, u_{2}\right) \in \partial V \text { and } \mu \geq 0 .
$$

If not, there exist $\left(u_{1}, u_{2}\right) \in \partial V$ and $\mu \geq 0$ such that $\left(u_{1}, u_{2}\right)=T\left(u_{1}, u_{2}\right)+\mu(e, e)$. Without loss of generality, we can assume that for all $t \in\left[a_{1}, b_{1}\right]$ we have

$$
\rho \leq u_{1}(t) \leq \rho / c, \quad \min _{t \in\left[a_{1}, b_{1}\right]} u_{1}(t)=\rho \quad \text { and } \quad 0 \leq u_{2}(t) \leq \rho / c .
$$

Then, for $t \in\left[a_{1}, b_{1}\right]$, we obtain

$$
\begin{aligned}
u_{1}(t) & =\lambda_{1} \int_{a}^{b} k_{1}(t, s) g_{1}(s) f_{1}\left(u_{1}(s), u_{2}(s)\right) d s+\mu e(t) \\
& \geq \lambda_{1} \int_{a_{1}}^{b_{1}} k_{1}(t, s) g_{1}(s) f_{1}\left(u_{1}(s), u_{2}(s)\right) d s+\mu \geq \lambda_{1} M \rho \gamma_{1, *}+\mu>\rho+\mu .
\end{aligned}
$$

Thus, we obtain $\rho=\min _{t \in\left[a_{1}, b_{1}\right]} u(t)>\rho+\mu \geq \rho$, a contradiction.

Therefore by Proposition 2.2 we have $i_{K}(T, V)=0$ and the proof is finished. 
Remark 3.2 The following condition, similar to the one given in [7], implies $\left(\mathrm{H}_{7}\right)$ and it is easier to check.

$\left(\mathrm{H}_{7}\right)^{*}$ For every $i=1,2, \lim _{u_{i} \rightarrow+\infty} \frac{f_{i}\left(u_{1}, u_{2}\right)}{u_{i}}=+\infty$, uniformly w.r.t. $u_{j} \in[0, \infty), j \neq i$.

Remark 3.3 In order to deal with negative kernels $k_{i}(t, s)<0$ we can require conditions $\left(\mathrm{H}_{2}\right),\left(\mathrm{H}_{3}\right)$, and $\left(\mathrm{H}_{5}\right)$ on the absolute value of the kernel such that $\left|k_{i}(t, s)\right|>0$ and conditions $\left(\mathrm{H}_{4}\right),\left(\mathrm{H}_{6}\right)$, and $\left(\mathrm{H}_{7}\right)$ on $\operatorname{sgn}\left(k_{i}\right) \cdot f_{i}$.

As an illustrative example, we apply our results to the system of ODEs

$$
\begin{aligned}
& u_{1}^{\prime \prime}(t)+\lambda_{1} f_{1}\left(u_{1}(t), u_{2}(t)\right)=0, \quad t \in(0,1), \\
& u_{2}^{\prime \prime}(t)+\lambda_{2} f_{2}\left(u_{1}(t), u_{2}(t)\right)=0, \quad t \in(0,1),
\end{aligned}
$$

with the BCs

$$
\begin{array}{ll}
u_{1}^{\prime}(0)=0, & u_{1}(1)+u_{1}^{\prime}(1)=0, \\
u_{2}^{\prime}(0)=0, & u_{2}(1)=\xi u_{2}(\eta), \quad \eta, \xi \in(0,1) .
\end{array}
$$

To the system (3.4)-(3.5) we associate the system of integral equations

$$
\begin{aligned}
& u_{1}(t)=\lambda_{1} \int_{0}^{1} k_{1}(t, s) f_{1}\left(u_{1}(s), u_{2}(s)\right) d s, \\
& u_{2}(t)=\lambda_{2} \int_{0}^{1} k_{2}(t, s) f_{2}\left(u_{1}(s), u_{2}(s)\right) d s,
\end{aligned}
$$

where the Green's functions are given by

$$
k_{1}(t, s)= \begin{cases}2-t, & s \leq t \\ 2-s, & s>t\end{cases}
$$

and

$$
k_{2}(t, s)=\frac{1}{1-\xi}(1-s)-\left\{\begin{array}{ll}
\frac{\xi}{1-\xi}(\eta-s), & s \leq \eta, \\
0, & s>\eta
\end{array}- \begin{cases}t-s, & s \leq t \\
0, & s>t .\end{cases}\right.
$$

The Green's function $k_{1}$ was studied in [28] were it was shown that we may take (with our notation)

$$
\Phi_{1}(s)=(2-s), \quad \gamma_{1}^{*}=\frac{3}{2} .
$$

The choice of $\left[a_{1}, b_{1}\right]=[0,1]$ gives

$$
c_{1}=\frac{1}{2}, \quad \gamma_{1, *}=1
$$


The kernel $k_{2}$ was extensively studied in $[28,29]$ and is more complicated to be dealt with, due to the presence of the nonlocal term in the BCs. In this case we may take

$$
\Phi_{2}(s)=k_{2}(0, s)=\left\{\begin{array}{ll}
\frac{1-s}{1-\xi}, & \text { if } \eta<s \leq 1, \\
\frac{1-s-\xi(\eta-s)}{1-\xi}, & \text { if } 0 \leq s \leq \eta,
\end{array} \quad \gamma_{2}^{*}=\frac{1-\xi \eta^{2}}{2(1-\xi)} .\right.
$$

The choice, as in $[29]$, of $\left[a_{2}, b_{2}\right]=\left[0, b_{2}\right]$, where

$$
b_{2}= \begin{cases}\frac{1-\xi \eta}{2(1-\xi)}, & \text { if } 1+\xi \eta \leq 2 \eta, \\ \frac{1}{(2-\xi)}, & \text { if } 1+\xi \eta>2 \eta,\end{cases}
$$

leads to

$$
c_{2}=\frac{1-\xi \eta-(1-\xi) b_{2}}{1-\xi \eta}, \quad \gamma_{2, *}= \begin{cases}b_{2}^{2}, & \text { if } 1+\xi \eta \leq 2 \eta, \\ \frac{1-2 \xi \eta^{2}+\xi^{2} \eta^{2}}{2(1-\xi)(2-\xi)}, & \text { if } 1+\xi \eta>2 \eta\end{cases}
$$

We now fix, as in [28], $\eta=1 / 2, \xi=1 / 4$. This gives $b_{2}=4 / 7$ and

$$
\gamma_{2}^{*}=\frac{5}{8}, \quad c_{2}=\frac{25}{49}, \quad \gamma_{2, *}=\frac{19}{56} .
$$

Furthermore take

$$
f_{1}\left(u_{1}, u_{2}\right)=\left(2+\sin \left(u_{2}\right)\right) u_{1}^{2}, \quad f_{2}\left(u_{1}, u_{2}\right)=\left(2+\sin \left(u_{1}\right)\right) u_{2}^{2} .
$$

In the case of the nonlinearities (3.9), we can choose $B_{1}=B_{2}=\pi / 2$. We observe that condition $\left(\mathrm{H}_{7}\right)^{*}$ holds, we note that $c=\min \left\{c_{1}, c_{2}\right\}=1 / 2$ and that

$$
\sup _{r_{1} \in(0, \pi / 2), r_{2} \in(0, \pi / 2)} \frac{r_{i}}{2 f_{i}\left(r_{1}, r_{2}\right) \gamma_{i}^{*}}=+\infty, \quad \text { for every } i .
$$

As a consequence, by means of Theorem 3.1, we obtain a non-zero solution of the system (3.4)-(3.5) for every $\lambda_{1}, \lambda_{2} \in(0, \infty)$.

\section{Competing interests}

The authors declare that they have no competing interests.

\section{Authors' contributions}

All the authors contributed equally and significantly in writing this article. All the authors read and approved the final manuscript.

\section{Author details}

'Departamento de Análise Matemática, Facultade de Matemáticas, Universidade de Santiago de Compostela, Santiago de Compostela, 15782, Spain. ²Departamento de Matemáticas, Universidade de Vigo, Pabellón 3, Campus de Ourense, Ourense, 32004, Spain. ${ }^{3}$ Dipartimento di Matematica e Informatica, Università della Calabria, Arcavacata di Rende, Cosenza, 87036, Italy.

\section{Acknowledgements}

The authors would like to thank the anonymous referee for his/her valuable comments, which have improved the correctness and the presentation of the manuscript. A Cabada was partially supported by Ministerio de Educación y Ciencia, Spain, and FEDER, Projects MTM2010-15314 and MTM2013-43014-P, JA Cid was partially supported by Ministerio de Educación y Ciencia, Spain, and FEDER, Project MTM2013-43404-P and G Infante was partially supported by G.N.A.M.P.A. - INdAM (Italy). This paper was partially written during a visit of G Infante to the Departamento de Análise Matemática of the Universidade de Santiago de Compostela. G Infante is grateful to the people of the aforementioned Departamento for their kind and warm hospitality. 


\section{References}

1. Cabada, A, Cid, JA: Existence of a non-zero fixed point for non-decreasing operators via Krasnoselskii's fixed point theorem. Nonlinear Anal. 71, 2114-2118 (2009)

2. Cabada, A, Cid, JA, Infante, G: New criteria for the existence of non-trivial fixed points in cones. Fixed Point Theory Appl. 2013, 125 (2013)

3. Cid, JA, Franco, D, Minhós, F: Positive fixed points and fourth-order equations. Bull. Lond. Math. Soc. 41, 72-78 (2009)

4. Franco, D, Infante, G, Perán, J: A new criterion for the existence of multiple solutions in cones. Proc. R. Soc. Edinb., Sect. A 142, 1043-1050 (2012)

5. Persson, H: A fixed point theorem for monotone functions. Appl. Math. Lett. 19, 1207-1209 (2006)

6. Agarwal, RP, O'Regan, D, Wong, PJY: Constant-Sign Solutions of Systems of Integral Equations. Springer, Cham (2013)

7. Cheng, $X, Z$ hang, Z: Existence of positive solutions to systems of nonlinear integral or differential equations. Topol. Methods Nonlinear Anal. 34, 267-277 (2009)

8. Cheng, X, Zhong, C: Existence of positive solutions for a second-order ordinary differential system. J. Math. Anal. Appl. $312,14-23(2005)$

9. Dunninger, DR, Wang, H: Existence and multiplicity of positive solutions for elliptic systems. Nonlinear Anal. 29, 1051-1060 (1997)

10. Dunninger, DR, Wang, H: Multiplicity of positive radial solutions for an elliptic system on an annulus. Nonlinear Anal. 42, 803-811 (2000)

11. Franco, D, Infante, G, O'Regan, D: Nontrivial solutions in abstract cones for Hammerstein integral systems. Dyn. Contin. Discrete Impuls. Syst., Ser. A Math. Anal. 14, 837-850 (2007)

12. Goodrich, CS: Nonlocal systems of BVPs with asymptotically superlinear boundary conditions. Comment. Math. Univ. Carol. 53, 79-97 (2012)

13. Goodrich, CS: Nonlocal systems of BVPs with asymptotically sublinear boundary conditions. Appl. Anal. Discrete Math. 6, 174-193 (2012)

14. Henderson, J, Luca, R: Existence and multiplicity for positive solutions of a system of higher-order multi-point boundary value problems. NoDEA Nonlinear Differ. Equ. Appl. 20, 1035-1054 (2013)

15. Henderson, J, Luca, R: Positive solutions for systems of second-order integral boundary value problems. Electron. J. Qual. Theory Differ. Equ. 2013, 70 (2013)

16. Infante, G, Pietramala, P: Existence and multiplicity of non-negative solutions for systems of perturbed Hammerstein integral equations. Nonlinear Anal. 71, 1301-1310 (2009)

17. Karakostas, GL: Existence of solutions for an n-dimensional operator equation and applications to BVPs. Electron. J. Differ. Equ. 2014, 71 (2014)

18. Lan, KQ: Multiple positive solutions of semilinear differential equations with singularities. J. Lond. Math. Soc. 63 , 690-704 (2001)

19. Lan, KQ, Lin, W: Multiple positive solutions of systems of Hammerstein integral equations with applications to fractional differential equations. J. Lond. Math. Soc. 83, 449-469 (2011)

20. Lan, KQ, Lin, W: Positive solutions of systems of singular Hammerstein integral equations with applications to semilinear elliptic equations in annuli. Nonlinear Anal. 74, 7184-7197 (2011)

21. Yang, Z: Positive solutions for a system of nonlinear Hammerstein integral equations and applications. Appl. Math. Comput. 218, 11138-11150 (2012)

22. Yang, Z, Zhang, Z: Positive solutions for a system of nonlinear singular Hammerstein integral equations via nonnegative matrices and applications. Positivity 16, 783-800 (2012)

23. Goodrich, CS: On nonlinear boundary conditions involving decomposable linear functionals. Proc. Edinb. Math. Soc. (2014). doi:10.1017/S0013091514000108

24. Zeidler, E: Nonlinear Functional Analysis and Its Applications. I: Fixed-Point Theorems. Springer, New York (1986)

25. Amann, $\mathrm{H}$ : On the number of solutions of nonlinear equations in ordered Banach spaces. J. Funct. Anal. 11, 346-384 (1972)

26. Amann, H: Fixed point equations and nonlinear eigenvalue problems in ordered Banach spaces. SIAM Rev. 18 620-709 (1976)

27. Guo, D, Lakshmikantham, V: Nonlinear Problems in Abstract Cones. Academic Press, Boston (1988)

28. Webb, JRL: A class of positive linear operators and applications to nonlinear boundary value problems. Topol. Methods Nonlinear Anal. 39, 221-242 (2012)

29. Webb, JRL: Remarks on positive solutions of three point boundary value problems. In: Proceedings of the Fourth International Conference on Dynamical Systems and Differential Equations (Wilmington, NC, 2002). Discrete Contin. Dyn. Syst., suppl., pp. 905-915 (2003)

doi:10.1186/s13661-014-0254-8

Cite this article as: Cabada et al.: A positive fixed point theorem with applications to systems of Hammerstein

integral equations. Boundary Value Problems 2014 2014:254 